\title{
Life Trend Analysis of Aircraft's Key Component Based on Power Spectral Envelope Energy and SVM
}

\author{
Jianguo Cui ${ }^{1}$, Baosheng $\mathrm{Liu}^{2}$, Guihua $\mathrm{Wang}^{3}$, Mingyue $\mathrm{Yu}^{4}$ and Yang Gao ${ }^{5}$ \\ 1,2,4 Shenyang Aerospace University \\ ${ }^{3,5}$ Shenyang Institute of engine design \\ 'gordon_cjg@163.com,2514156430@qq.com,3469866206@qq.com, \\ ${ }^{4} 1498245199 @ q q . c o m, 53071844681 @ q q . c o m$
}

\begin{abstract}
The life prediction method based on the power spectral envelope energy and Support Vector Machine (SVM), which is the combination of time-domain and frequency-domain, is proposed for the key component of aircraft's (aircraft air refrigerator) life unpredictable problems. By first, made a long-term test for aircraft air refrigerator through aircraft-specific life testing platform, then got a lot of acceleration parameters which characterize the health status of the aircraft air refrigerator. Through the analysis of acceleration parameters' power spectrum, its power spectrum envelope energy would be obtained. Used the power spectrum envelope energy to construct feature vector, and designed SVM life prediction model and using this model to predict aircraft air refrigerator's lifetime. The research results show that the life prediction method based on the power spectral envelope energy and SVM can achieve a good life prediction of aircraft air refrigerator, and laid the foundation for life prediction of air refrigerator in aircraft engineering application.
\end{abstract}

Keywords: Aircraft Air Refrigerator; Power Spectrum Envelope Energy; SVM; Life; Predict

\section{Introduction}

Aircraft air refrigerator is one of the key components of the aircraft's air conditioning system [1], and its working state directly affects the permitted temperature range of the pilot and electronic devices. Once the air refrigerator's function is failure during the flight of plane, this is bound to affect the normal operation of electronic equipment and pose a great threat to the safe operation of the aircraft. So life prediction of aircraft air refrigerator has a very important significance and research value.

Currently, air refrigerator temperature difference (difference between inlet temperature and outlet temperature) or expansion ratio (ratio of the inlet pressure and the outlet pressure) is directly seen as parameters to characterize their health status in many documents during the research on life prediction of aircraft air refrigerator. But these parameters can suddenly change and cause a big impact on forecast accuracy at the moment of air refrigerator failure. So, In order to more accurately forecast air refrigerator's life, the life prediction method based on the power spectral envelope energy and Support Vector Machine (SVM) is proposed. Firstly, find out characteristics when the air refrigerator is failure by means of the analysis on the frequency-domain, treating these characteristics as a basis for

Foundation item: Aeronautical Science Foundation of China (No. 2010ZD54012); National Defence Pre-research Foundation (No. A0520110023); Defense Industrial Technology Development Program (No. Z052012B002); Simulation technology research of life test and storage quality of UAV-1(No.XY201434-1) 
follow-up time-domain forecast and using SVM model to predict the life of the aircraft air refrigerator.

\section{Life Prediction Theories}

\subsection{The Power Spectral Envelope Energy}

Power spectrum is an effective way to describe the product of random vibration. In fact, it is translated the original description of the vibration in the time domain into the description of the vibration in frequency domain [2]. It can be better extract the signal characteristic. If ${ }^{u(t)}$ is a function that can be performed the Fourier transform, then:

$$
u(v)=\int_{-\infty}^{\infty} u(t) e^{-i 2 \pi v t} d t
$$

Because $^{u(t)}$ is a real function, in general ${ }^{u(v)}$ is a complex function, they meet Barcelona cutting formula:

$$
\int_{-\infty}^{\infty} u^{2}(t) d t=\int_{-\infty}^{\infty}|u(v)|^{2} d v
$$

If ${ }^{u(t)}$ as for vibration acceleration, then the left side of the equation represents the total energy of vibration ${ }^{u(t)}$ on an infinite range. Integral on the right side is called the energy spectral density function. It is a non-negative real number, which means that the energy in unit frequency.

In practice and from the mathematical sense, most of ${ }^{u(t)}$ cannot be Fourier transformed. If use the truncated function $u T(t)=\left\{\begin{array}{l}u(t),|t| \leq T \\ 0,|t|>T\end{array}\right.$ to intercept ${ }^{u(t)}$, then for a limited duration truncated function ${ }^{u T(t)}$ can be Fourier transformed.

$$
u T(v)=\int_{-\infty}^{\infty} u T(t) e^{i 2 \pi v t} d t=\int_{-T}^{T} u(t) e^{i 2 \pi v t} d t
$$

Likewise, it also satisfies the Barcelona cutting formula,

$$
\int_{-\infty}^{\infty} u^{2} T(t) d t=\int_{-\infty}^{\infty}|u T(v)|^{2} d v
$$

Contact the truncated function, both ends of the equation is divided by $2 \mathrm{~T}$ and let $\mathrm{T}$ tends to infinity, then:

$$
\lim _{r \rightarrow \infty} \frac{1}{2 T} \int_{-T}^{T} u^{2}(t) d t=\int_{-\infty}^{\infty} \lim _{T \rightarrow \infty} \frac{1}{2 T}|u T(v)|^{2} d v
$$

Correspond to the definition of power spectral density, the right side of (5) is called the power spectral density [3], referred to the power spectrum, and denoted as fallows.

$$
P(v)=\lim _{T \rightarrow \infty} \frac{|u T(v)|^{2}}{2 T}
$$

The power spectrum from the visible expression is a non-negative real number. It is a volume in the frequency domain, which we can see from the derivation of the power spectrum, and it directly responses the values at different frequencies corresponding to the frequency domain. 
Determine the local maxima of the power spectrum ${ }^{P(v)}$, the maximum points connected by a cubic spline, to get the power spectral envelope. If $|u(t)|^{2}$ as to the energy density of the signal, then the power spectral envelope energy is:

$$
E(v)=\int_{-\infty}^{\infty}|p(v)|^{2} d v
$$

\subsection{Support Vector Machine}

SVM is a machine learning method based on statistical learning theory, which can well solve the small sample, high dimension, over learning and local minimum problems. It provides an effective solution for the prediction of time series [4]. The basic idea of SVM to predict is that through a nonlinear mapping ${ }^{\varphi}$ to map the data $X_{i}$ into a high dimensional feature space $\mathrm{F}$, and liner regression in this space, which will transform the non-liner regression problem of low-dimensional feature into a high dimensional feature space liner regression problem to solve. Regression function determined by the statistical learning theory is as follows:

$$
f(x)=(w, \varphi(x))+b
$$

In which, ${ }^{\varphi}: \mathrm{R}^{m} \rightarrow \mathrm{F}, \omega \in F,{ }^{\varphi}$ is the nonlinear mapping from space $R^{m}$ to space F. $X \in R^{m}, \quad(w, \varphi(x))$ represents the inner product of $\mathrm{W}$ and ${ }^{\varphi(x)}, \mathrm{W}$ is the weight vector, $\omega \in F, b$ is the bias.

The traditional solution is to find a regression function $\mathrm{f}$, make the empirical risk minimization. SVM regression problem is solved by making minimal of the experience risk and confidence risk, then the prediction model has a very good function of approximation ability and generalization ability[5]. In (8), ${ }^{\varphi(x)}$ is known, use the sample data $\left({ }^{X_{i}}, Y_{i}\right)$, by the following functional minimization can be obtained the estimated values of $\mathrm{w}$ and $\mathrm{b}$ in (8).

$$
R_{r e s}[f]=R_{e m p}[f]+\lambda\left\|_{w}\right\|^{2}=\sum_{i=1}^{s} C\left(\mathrm{e}_{i}\right)+\lambda\|\omega\|^{2}
$$

In which, ${ }^{R_{\text {emp }}[f]}$ is the empirical risk, $\|\omega\|^{2}$ is the confidence risk. ${ }^{C\left(\mathrm{e}_{i}\right)}$ is the experience loss of the model, ${ }^{C(\cdot)}$ is the loss function, ${ }_{i}=f\left(X_{i}\right)-Y_{i}=\hat{Y}_{i}-Y_{i}$ is the difference between the sample value of the predicted and the true value, $S$ is the sample size.

Solving (9) is equivalent to solving the following optimization problem:

$$
\begin{array}{r}
\min L=\frac{1}{2} w^{T} w+C \sum_{i=1}^{s}\left(\xi_{i}^{*}+\xi_{i}\right) \\
\text { s.t. }\left\{\begin{array}{c}
y_{i}-\left(w, \varphi\left(X_{i}\right)\right)-b \leq \varepsilon+\xi_{i}^{*} \\
\left.\left(w, X_{i}\right)\right)+b-y_{i} \leq \varepsilon+\xi_{i}
\end{array}\right. \\
\xi_{i}, \xi_{i}^{*} \geq 0
\end{array}
$$


In which, $C=\frac{1}{\lambda}, \xi_{i}$ and $\xi_{i}^{*}$ are Lagrange multipliers. To facilitate solving, transform the quadratic programming problem to its dual problem.

$$
\begin{aligned}
\max J= & -\frac{1}{2} \sum_{i, j=1}^{s}\left(\alpha_{i}-\alpha_{i}\right)\left(\alpha_{j}-\alpha_{j}^{j}\right)\left(\varphi\left(X_{i}\right), \varphi\left(X_{j}\right)\right) \\
& +\sum_{i=1}^{s} \alpha_{i}\left(Y_{i}-\varepsilon\right)-\sum_{i=1}^{s} \alpha_{i}\left(Y_{i}+\varepsilon\right) \\
& \left\{\begin{array}{l}
s \\
\sum_{i=1} \alpha_{i}=\sum_{i=1}^{s} \alpha_{i}
\end{array}\right. \\
\text { s.t. }\left\{\begin{array}{l}
0 \leq \alpha_{i} \leq C \\
\end{array}\right. & \mid 0 \leq \alpha_{i}^{*} \leq C
\end{aligned}
$$

The result is ${ }^{w=\sum_{i=1}^{s}\left(\alpha_{i}-\alpha_{i}^{*}\right) \varphi\left(X_{i}\right)}$, "b" can be got by any one of the support vectors into the evaluated, and then the non-linear function ${ }^{f}$ will get.

$$
f(x)=\sum_{i=1}^{s}\left(\alpha_{i}-\alpha_{i}^{*}\right)\left(\varphi\left(X_{i}\right), \varphi(X)\right)+b
$$

Define the high-dimensional feature space transformation inner product operation for kernel function of SVM [6]. ${ }^{K\left(X_{i}, X\right)=\left(\varphi\left(X_{i}\right), \varphi(X)\right)}$, Only the variables in the original low dimension doing kernel function operation can get its inner product in high dimensional space, then nonlinear mapping of solving convex quadratic programming problems is:

$$
f(x)=\sum_{i=1}^{l}\left(\alpha_{i}-\alpha_{i}^{*}\right)\left(K\left(X_{i}, X\right)+b\right.
$$

\section{Life Prediction Model for aircraft air refrigerator}

In order to accurately predict the effective life of the aircraft air refrigerator, it should be chosen the parameters that best characterize the air refrigerator's health status, and on parameters for efficient feature extraction, then use the effective feature extraction results to the model for prediction. Finally get the remaining life of the aircraft air refrigerator. Specific steps are as follows:

Step 1: Select parameters which could the best characterize the health status of the aircraft air refrigerator, denoted as $u(t)$. Fourier transform to it, and by (6) obtained the power spectral $P(v)$ of $u(t)$.

Step 2: The power spectral $P(v)$ is non-negative real number, determine its local maxima, with a cubic spline lines connecting the maxima, then obtained power spectral envelope. By (7) obtained the envelope energy $E(v)$ of the power spectral.

Put the power spectral envelope energy $E(v)$ as characteristic sequence to predict the life of aircraft air refrigerator. Contrast the $E(v)$ change of aircraft air refrigerator in normal operation and function failure, effectively extract the feature information of their health status. 
Step 3: Reconstruct phase space of the power spectral envelope energy sequence, train the SVM model and predict the life of the aircraft air refrigerator., there are three most commonly used SVM kernel function are as follows:

Polynomial kernel: $K\left(X, X_{i}\right)=\left(\left(X, X_{i}\right)+t\right)^{q}, t>0, q \in N$

RBF kernel: $K\left(X, X_{i}\right)=\exp \left(-\frac{\left\|X-X_{i}\right\|^{2}}{2 \sigma^{2}}\right), \sigma>0$

Hyperbolic kernel: $K\left(X, X_{i}\right)=\tanh \left(a\left(X, X_{i}\right)+t\right), a, t$ is constant.

Step 4: Put the failure time in actual test corresponding power spectral envelope energy as the lifetime threshold of aircraft air refrigerator. In order to determine the validity of the model compared the lifetime ${ }^{T_{1}}$ of air refrigerator which predicted by the SVM model and the lifetime ${ }^{T_{2}}$ of the real test and according to (14) calculate the relative error.

$$
w=\frac{\left|T_{1}-T_{2}\right|}{T_{2}} \times 100 \%
$$

\section{Research on Experimental Verification}

\subsection{Acquisition and Pre-Processing of a Certain Type of Aircraft Air Refrigerator Life Information}

For a certain type of aircraft air refrigerator, do accelerated life test relying on a dedicated test platform for its life prediction. Through the test, get a lot of whole life characterization parameters of air refrigerator, including acceleration, speed, inlet pressure, outlet pressure, inlet temperature, inlet flow, outlet temperature, outlet flow and so on. As air refrigerator is rotating machinery, so make the radial acceleration as the research object for the air refrigerator's life trend analysis and forecasting, which can best reflect the air refrigerator's vibration. The original radial accelerations got in the test are shown in Figure 1.

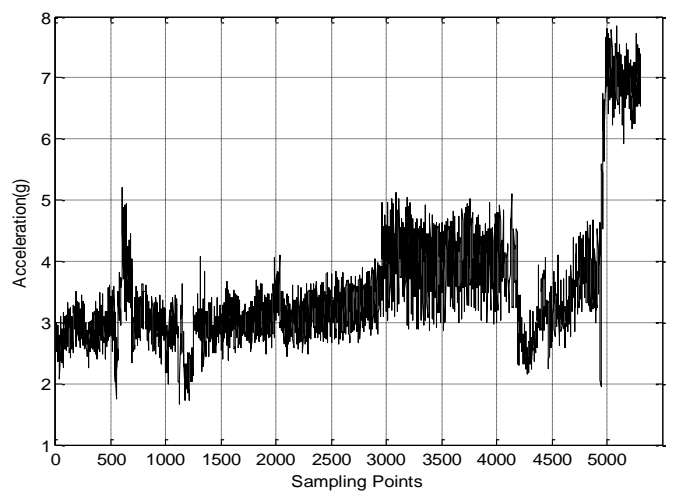

Figure 1. The Original Radial Accelerations

In order to find a better life change trend, pretreated the original acceleration information before, and removed significantly interfere fluctuation data during the test.

\subsection{Power Spectral Envelope Energy}

Calculate the power spectral envelope energy of aircraft air refrigerator's radial acceleration. First of all, dynamic grouping the original valid acceleration: fixed the number of acceleration data is 300 in each group, the first group have 300 acceleration 
data, then slide backwards of 30 data, which constitute the second group, using the same method, each time slide backwards of 30 acceleration data, finally received 131 groups of full lifetime data of aircraft air refrigerator. Fourier transform for each group of data respectively, and through (6) to calculate the power spectrum. The power spectrum of the aircraft air refrigerator under the normal operation are basically identical, represented by the first group of power spectrum, as shown in Figure 2; similarly, the power spectrum of the air refrigerator in failure state is shown in Figure 3.

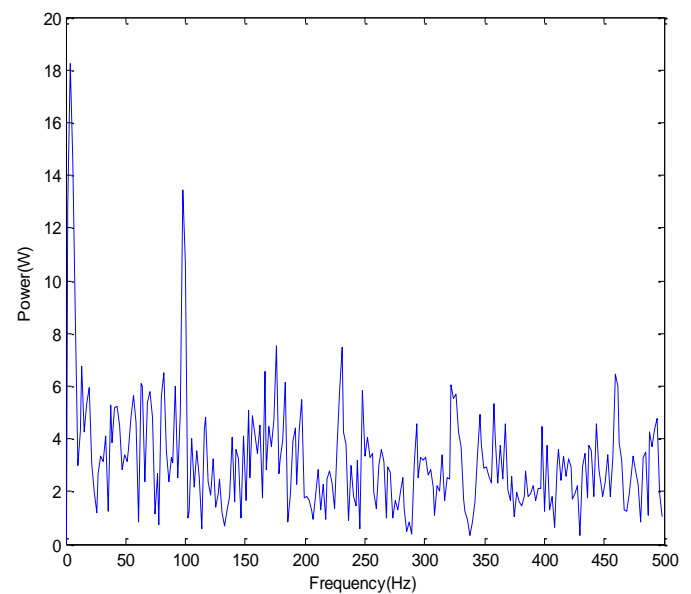

Figure 2. The Power Spectrum of Air Refrigerator under the Normal Operation

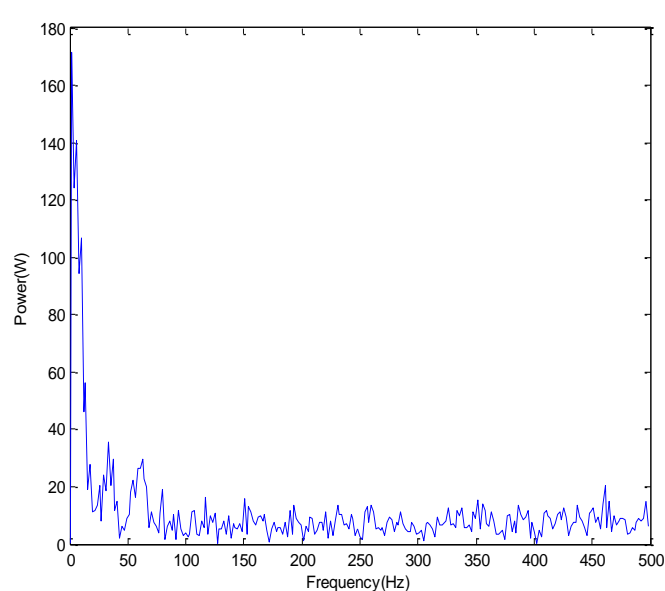
Figure 3. The Power Spectrum of Air Refrigerator under Function Failure

Comparing Figure 2 and Figure 3 shows, from normal operation to the function fails, the power spectrum of aircraft air refrigerator is obvious different. Under normal operation condition, when ${ }^{f=100 \mathrm{~Hz}}$, it has obvious peak in the power spectrum, this frequency represents the normal operation of the aircraft air refrigerator's natural frequency when it operate normally; when ${ }^{f=100 \mathrm{~Hz}}$, the function of the air refrigerator was failure, its power spectrum is flat, which means the inherent frequency as the failure of air refrigerator has been lost. This phenomenon shows that the power spectrum analysis method can effectively extract the characteristics of the aircraft air refrigerator's health status.

As for the dynamic grouping of the acceleration, it makes the number of power spectrum is more and analysis is more complicated. Therefore, it needed to further extract characteristic of the power spectrum. Respectively using (11) for each power spectrum, and then got the power spectral envelope energy, the power spectral envelope energy are shown in Figure 4, a total of 131 data, and each power spectral envelope energy representative within five minutes of health information. In Figure 4, the 123th point is corresponds to the function failure point in the actual test of aircraft air refrigerator. Therefore, regard the acceleration's power spectral envelope energy equal to 50 as the lifetime threshold line, when the power spectral envelope energy more than 50, it means the aircraft air refrigerator function failure. Finally, used the power spectral envelop energy as feature sequence to predict, then can obtain the predicted remaining life of aircraft air refrigerating. 


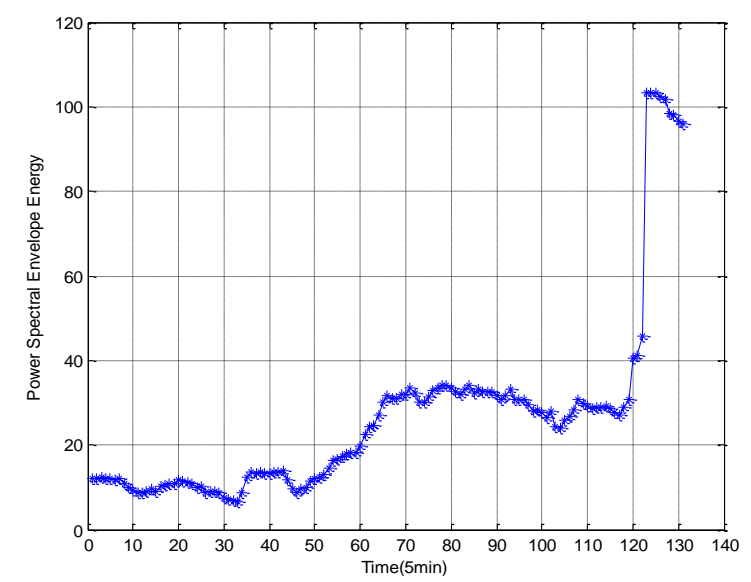

Figure 4. The Power Spectral Envelope Energy of the Acceleration

\subsection{The Test of Life Prediction Based on SVM}

Make the power spectral envelope energy value of radial acceleration as a characteristic sequence, using the SVM model for prediction of air refrigerator life of aircraft. Specific steps are as follows:

Step 1: By phase space reconstruction of the power spectral envelope energy sequence, and then get the input samples and output samples of SVM model.

Since the power spectral envelope energy value was a single variable sequence, while SVM model is suitable for multivariable prediction, then need the power spectral envelope energy sequence to phase space reconstruct. The principle of phase space reconstruction is to control the variable number of phase space reconstruction, that is to say, using the sequence of $1 \sim n$ to predict the number of $n+1$, using the sequence of $2 \sim n+1$ to predict the number of $n+2$, and so on, then can construct the input samples and output samples of SVM.

The test was obtained 131 power spectral envelope energy values, for easy to predict, make the variable number of phase space reconstruction $n=5$. That is, using the sequence of the first five to predict the sixth value, using the sequence of 2-6 to predict the seventh value, according to this method, till using the sequence of 126-130 to predict the value of 131th, specific as shown in Table 1.

\section{Table 1. Input and Output Sample Initially Constructed of the SVM Model}

\begin{tabular}{cc}
\hline Input samples $x$ & Output samples $y$ \\
\hline $1 \sim 5$ & 6 \\
$2 \sim 6$ & 7 \\
$\vdots$ & $\vdots$ \\
$125 \sim 129$ & 130 \\
$126 \sim 130$ & 131 \\
\hline
\end{tabular}

We can see from Table 1, the dimension of input samples is $126 \times 5$, the dimension of the output samples is $126 \times 1$. Due to the result in test of the 123th power spectral envelope energy point corresponding to the actual function of the failure point, in order to sample the input and output samples closer to the actual test, repeating the five sets of relatively flat power spectrum envelope of the input energy, then the dimension of SVM input samples is $131 \times 5$, the dimension of the output samples is $131 \times 1$, and the 123 th point corresponding the output sample point of failure, as shown in Table 2. 
Table 2. The Input and Output Sample of SVM

\begin{tabular}{|c|c|}
\hline Input samples $x$ & Output amples $y$ \\
\hline $1 \sim 5$ & 6 \\
\hline$\vdots$ & $\vdots$ \\
\hline $5 \sim 9$ & 10 \\
\hline $1 \sim 5$ & 6 \\
\hline$\vdots$ & $\vdots$ \\
\hline $118 \sim 122$ & 123 \\
\hline$\vdots$ & $\vdots$ \\
\hline $125 \sim 129$ & 130 \\
\hline $126 \sim 130$ & 131 \\
\hline
\end{tabular}

Step 2: Determine the kernel function of SVM, and create the training set and test set of SVM.

Through the study of three kinds of kernel functions commonly used in SVM model, concluded that the radical basis kernel function best, so using it in the SVM model. After several tests, decide to use the first 20 groups as training sample for SVM model, the rest of the groups as test sample, to verify the validity of SVM model.

Step 3: Use the SVM model to predict the life of aircraft air refrigerator.

The result of aircraft air refrigerator's life prediction based on SVM model is shown in Figure 5, the relative error of the prediction is $7.29 \%$.

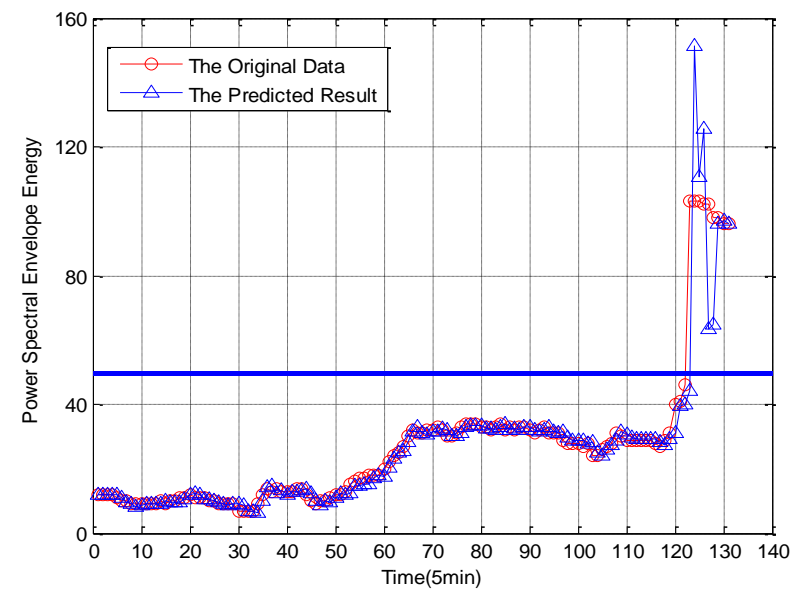

Figure 5. The Result of Aircraft Air Refrigerator's Life Prediction Based on SVM

As shown in Figure 5, the power spectrum envelope energy $E(v)=50$ is the lifetime threshold of the aircraft air refrigerator, when the prediction exceeds 50 , the air refrigerator is deactivated. From Figure 5 we can learn, at the 125 th point, $E(v)>50$, since each point represents $5 \mathrm{~min}$ information, then predict the remaining life of aircraft air refrigerator is $T_{1}=\frac{125 \times 5 \mathrm{~min}}{60 \mathrm{~min}}=10.42 \mathrm{~h}$.

Step 4: According to the relative error, to determine the validity of the model.

Based on the SVM model obtained the remaining life of the air refrigerator $T_{1}=10.42 \mathrm{~h}$, in the test the real life $T_{2}=10.33 \mathrm{~h}$, according to equation (14) can find its corresponding error

$$
w=\frac{|10.42-10.33|}{10.33} \times 100 \%=0.87 \%
$$


The relative error of aircraft air refrigerator between predicted life and real life is $0.87 \%$. We can see that the life prediction model designed for aircraft air refrigerator is feasible and effective.

\section{Conclusion}

In this paper, the life prediction method based on the power spectral envelope energy and SVM is proposed for aircraft air refrigerator's life unpredictable problems. Treating the vibration acceleration as an important parameter for the characterization of air refrigerator's health status and using SVM model to predict the life of aircraft air refrigerator. The research shows that the relative error between predicted lifetime and actual lifetime is only $0.87 \%$. So, the life prediction method based on the power spectral envelope energy and SVM can achieve a good life prediction of aircraft air refrigerator and has good applications prospects.

\section{References}

[1] S. Lin, K. Xia, J. Li, Y. Sun, Y. Hou and C. Chen, "Application of air refrigerator in aircraft air condition system", Fluid machinery, vol. 32, no. 10, (2004)

[2] J. Lu Ren, “Air refrigerator", Refrigeration and air conditioning, vol. 8, no. 6, (2008), pp. 15-21.

[3] R. Zhang, B. Cai, C. Yang, Q. Xu and Y. Gu, "Numerical method of the power spectral density", High power laser and particle beams, vol. 12, no. 6, November (2000), pp. 661-664.

[4] B. Li, "The effective tool of vibration analysis - power spectral density", Packaging engineering, vol. 25, no. 3, (2004), pp. 46-50.

[5] Y. Han, T. Zhong and L. Shi, "Rolling bearing fault diagnosis based on envelope spectrum", Mechanical Research \& Application, (2008), pp. 118-119.

[6] W. Chen and Z. Fang, "Statisical characteristics of the envelope of a random signal and computation of the envelope spectrum", Shanghai journal of mechanics, vol. 17, no. 4, (1996) December, pp. 326-333.

[7] W. Qu, G. Fan and B. Yang, "Research on complicated time series prediction based on support vector machines", Computer engineering, vol. 31, no. 23, (2005) December, pp. 1-3.

[8] G. Xu and Y. Jia, "MATLAB implementation of pattern recognition and intelligent computing", Beijing: Beihang University Press, (2012), pp. 125-129.

[9] X. Wang, F. Shi, L. Yu and Y. Li, "MATLAB neural network analysis of 43 cases", Beijing: Beihang University Press, (2013), pp. 137-143.

\section{Author}

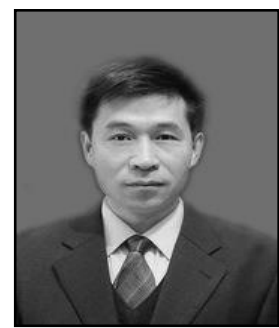

Jianguo Cui (1963-), male, Shenyang, Ph.D., professor, main research directions for vehicle health diagnosis, prediction and integrated health management, simulation technology and applications.

Email: gordon_cjg@163.com

Tel: 13082410088 
International Journal of Advanced Science and Technology Vol.84 (2015) 